\title{
Ultrafast Through-Space Electronic Energy Transfer in Molecular Dy- ads Built around Dynamic Spacer Units
}

\author{
Raymond Ziessel, ${ }^{\mathrm{a}}$ Patrycja Stachelek, ${ }^{\mathrm{a}, \dagger}$ Anthony Harriman, ${ }^{\mathrm{a}, *}$ Gordon J. Hedley, ${ }^{\mathrm{b}, \dagger}$ Thomas Ro- \\ land, ${ }^{b}$ Arvydas Ruseckas ${ }^{\mathrm{b}}$ and Ifor D. W. Samuel ${ }^{\mathrm{b}, *}$ \\ ${ }^{a}$ Molecular Photonics Laboratory, School of Chemistry, Bedson Building, Newcastle University, Newcastle upon \\ Tyne, NE1 7 RU, United Kingdom and ${ }^{\mathrm{b}}$ Organic Semiconductor Centre, SUPA, School of Physics \& Astronomy, Phys- \\ ical Science Building, University of St. Andrews, North Haugh, St Andrews, KY16 9SS, United Kingdom.
}

\begin{abstract}
A pair of complimentary molecular dyads have been synthesized around a 1,2-diaminocyclohexyl spacer that itself undergoes ring inversion. Despite these conformational exchange processes, the donor and acceptor occupy quite restricted spatial regions and they are not interchangeable. The donor and acceptor pair comprise disparate boron dipyrromethene dyes selected to display favorable electronic energy transfer (EET). Steady-state emission spectroscopy confirms that through-space EET from donor to acceptor is almost quantitative, aided by the relatively short separations. Ultra-fast time-resolved fluorescence spectroscopy has allowed determination of the rates of EET for both dyads. Surprisingly, in view of the close proximity of donor and acceptor (center-to-center separations less than $20 \AA$ ), the EET dynamics are well accounted for in terms of the computed molecular conformations and conventional Förster theory. One dyad appears as a single family of conformations but EET for the second dyad corresponds to dual-exponential kinetics. In this latter case, an intramolecular hydrogen bond helps stabilize an open geometry wherein EET is relatively slow.
\end{abstract}

\section{INTRODUCTION}

The Förster theory underpinning through-space electronic energy transfer (EET) in the weak-coupling limit, developed originally in the late $1940^{\prime} \mathrm{s},{ }^{1}$ serves as a guide for designing, for example, innovative molecular devices, ${ }^{2}$ sensors, ${ }^{3}$ labels ${ }^{4}$ and machines. 5 The theory is used extensively in biochemistry for estimating topological parameters ${ }^{6-8}$ in cases where X-ray crystal structures are difficult to obtain. Several critical features of Förster theory, most notably the inverse separation distance to the power of $\operatorname{six}^{9,10}$ and the dependence on the spectral overlap integral," have been confirmed by experiment but certain aspects remain obscure. In part, the difficulty in applying Förster theory to molecular dyads comprising an energy-donor and an energy-acceptor comes about because of the dynamic nature of the entity under study. ${ }^{12}$ This situation becomes especially important ${ }^{13,14}$ when the donor-acceptor separation distance is comparable to the sum of the respective transition dipole moment vectors. Spacer groups used to position the donor and acceptor units are prone to out-of-plane bending motions 15-19 $^{-19}$ while there is a continual problem to identify transition dipole moment vectors ${ }^{20}$ for highly conjugated fluorophores. Attempts to modify the theory for weaklycoupled reactants, such as the extended dipole method, ${ }^{21-24}$ have proved to be useful tools but there are few molecular dyads that bring the reactants into close proximity (i.e., <20 $\AA$ ) without providing a through-bond conduit for fast EET. ${ }^{25,26}$
Many of the artificial analogues designed for through-space EET are based on the dendrimer motif where multiple chromophores are arranged in layers around a central unit. ${ }^{27-29}$ Other architectures include cyclic wheels, ${ }^{30} \mathrm{U}$-shaped arrangements ${ }^{31}$ and orthogonally spaced donor-acceptor pairs. ${ }^{16,32}$ There are few examples of linear molecular dyads displaying through-spaced EET without the complications of accompanying through-bond coupling. Notable among these latter systems are those based on carborane-based spacers.33,34 Many other systems do not favor detailed mechanistic examinations because of flexible connections that prevent establishing the molecular topology. $35-38$ To date, the shortest distances over which through-space EET has been compared with theoretical estimates remains at ca. $20 \AA \AA^{10,12,15,16,25,26}$

We now introduce two molecular dyads built from dissimilar boron dipyrromethene (BODIPY) dyes 39 that enable exploration of through-space EET. These new dyads are assembled around a 1,2-diaminocyclohexyl (DACH) spacer unit. The latter is well known ${ }^{40}$ in classical organic chemistry because of its conformational exchange and chirality. Thus, DACH gives rise to cis- and trans-conformers as outlined in Chart 1 where $\mathrm{D}$ and $\mathrm{A}$, respectively, refer to the energy-donor and energyacceptor required for intramolecular EET. In the trans-configuration, both reactants can be held at axial $(a, a)$ or equatorial $(e, e)$ sites which interconvert by way of ring inversion. This latter process does not convert the molecule to the corre- 
sponding cis-geometry. Now, the cis-conformer has one reactant held at an axial site and the other at an equatorial site, giving rise to the mixed a,e configuration. This species can transform to the corresponding e, $a$ species but must retain the overall cis-geometry. Although these various interconversions are likely to be fast, ${ }^{41}$ they cannot compete with rapid (i.e., sub-ns) EET from D to A; the rate of EET being dependent on the nature of the reactants and the molecular topology. ${ }^{42} \mathrm{We}$ now examine the dynamics of EET in these molecular dyads using ultra-fast fluorescence spectroscopy. The primary purpose of the investigation is to determine if the timescale for the intramolecular EET event is consistent with the geometry of the dyad. It will be appreciated that each dyad is likely to exist in a wide, and possibly bimodal, distribution of conformations. This situation presents an interesting challenge in terms of achieving a proper understanding of the EET mechanism.

Chart 1. Pictorial Representation of the Likely Conformations for the Cis and Trans Molecular Dyads Assembled Around the 1,2-Diaminocyclohexyl Scaffold. It is Considered that Conformational Exchange is Frozen on the Timescale of EET.

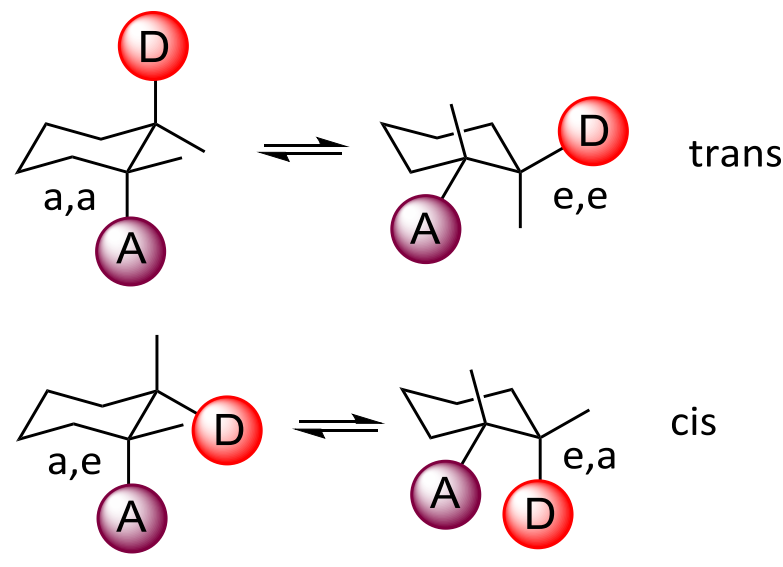

\section{RESULTS AND DISCUSSION}

\section{Materials}

The two new molecular dyads were built around the DACH spacer unit and their molecular formulas are given in Figure 1. In each case, the energy donor is formed from a regular boron dipyrromethene (BODIPY) dye,39 which is intended to function as the energy donor. Such dyes are usually equipped with fluorine atoms attached at the boron site but, to aid solubility, we have replaced fluorine with a short alkyne chain. 43 This strategy imports polarity to an otherwise nonpolar molecule and helps with chromatographic purification. The donor function is attached to the spacer by way of an amide linkage situated at the 4-position of a meso-phenyl ring. 44 Alkyl groups on the dipyrrin framework force the meso-phenyl ring into an almost orthogonal orientation that does not favor throughbond electronic interactions. Indeed, it has been demonstrated that the amide connection attached in this way is a poor conduit for electronic communication between terminal BODIPY residues. 44

The complementary acceptor needs to possess a somewhat lower excitation energy such that its absorption profile overlaps with fluorescence from the donor. This situation was achieved using a BODIPY derivative equipped 45 with a $\mathrm{N}, \mathrm{N}$ dimethylaminostyryl unit attached at the 3-position. The extra conjugation pushes the lowest-energy absorption transition to lower energy, as is required, and increases the corresponding oscillator strength. The amino group introduces polarity without the need to replace the B-F bonds. ${ }^{46}$ The asymmetric nature of this molecule complicates the synthesis but moving to the corresponding 3,5-bis-derivative decreases spectral overlap by a significant factor. Again, the acceptor unit is connected to the spacer via an amide group attached to the mesophenyl ring. The 1,7-methyl groups keep the meso-phenyl ring in a near orthogonal geometry and prevent the acceptor from operating as a molecular-scale rotor. 47

The choice of DACH as the spacer group was based on the perceived notion that closely-spaced dyads could be achieved. It was also realized that the cyclohexyl ring is structurally dynamic, even with large groups attached at either axial or equatorial sites. This situation leads to families of conformers rather than a single, well-defined entity. To enrich the diversity of these dyads, the amide linkages were attached at axial (a) or equatorial (e) sites so as to give axial-axial $(a, a)$ or axialequatorial $(a, e)$ geometries (Figure 1). Rapid equilibration should convert the $a, a$ conformer into the equatorial-equatorial $(e, e)$ conformer but the mixed $a, e$ conformer can only convert to the $e, a$ structure (Chart 1 ). In a crude sense, it is possible to consider these generic structures in terms of cis- and trans-geometries but it is important to specify the axial and equatorial distribution. The strategy used to realize these specific conformations involves a peptide coupling reaction between the commercially available cis- or trans-1,2-diaminocyclohexane primary amines and the corresponding BODIPY carboxylic acid derivatives mediated under ambient conditions by $\mathrm{O}-(\mathrm{N}-$ Succinimidyl)-N,N,N',N'-tetramethyluronium tetrafluoroborate (TSTU). ${ }^{8}$ The dedicated acid reagents were prepared according to a carboalkoxylation reaction using water as the nucleophile, carbon monoxide and the corresponding phenyliodo-BODIPY derivative. 49 The highly fluorescent donor (DON) and acceptor (ACC) derivatives were obtained as side-products during the peptide coupling reaction. The dimethylamino fragments arise from activation of the TSTU reagent. ${ }^{48,50}$ To aid the structural identification and to provide controls for the spectroscopic studies, a series of mono-chromophoric compounds was prepared (Figure 2). Full synthetic details are provided as part of the Supporting Information.

\section{Parameters for EET derived from control studies}

The simplest mono-chromophoric compounds used as controls for the spectroscopic studies have the donor (DON) or acceptor (ACC) functions terminated with an amide group. It was observed that replacing the fluorine atoms with the solubilizing alkyne residue had no effect on the photophysical properties recorded for the donor. To verify that the diaminocyclohexyl spacer unit does not enter into the light-induced processes, two more controls were introduced. These comprise one donor chromophore (DSp) or one acceptor unit (ASp) attached to the DACH spacer via an amide linkage. As indicated above, the DACH spacer can be resolved with the amino groups in what might be termed non-interconverting cis- and trans-isomers. This leads to four control compounds according to the geometry of the spacer (Figures 1 and 2). The photophysical properties of all the control compounds were established in 2-methyltetrahydrofuran (MTHF) solution at 
room temperature. Absorption and fluorescence spectra recorded at room temperature are shown primarily in the Supporting Information. A collection of the wavelengths corresponding to the absorption $\left(\lambda_{\mathrm{ABS}}\right)$ and emission $\left(\lambda_{\mathrm{FLU}}\right)$ maxima is provided in Table 1 . There is a significant difference in excitation energies $\left(E_{o o}\right)$, derived from the crossover points between normalized absorption and emission spectra, for donor and acceptor moieties but the presence of the spacer has negligible effect, thereby confirming its role as an innocent spectator.

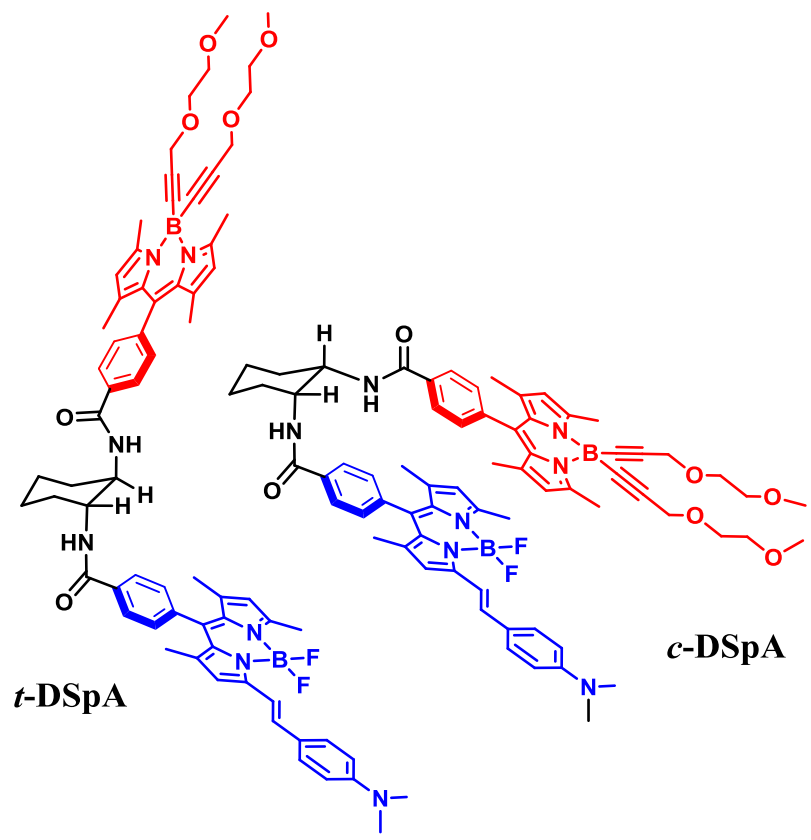

Figure 1. Representation of the target molecular dyads emphasizing the separation of donor (red) and acceptor (blue) moieties. These dyads are distinguished as possessing global trans-geometry (seen here on the left) or cis-geometry (seen here on the right) with respect to the spacer group. In crude terms, the cis-geometry positions both donor and acceptor on the same side of the DACH spacer while the trans-geometry places the chromophores on opposite side of the spacer

Also provided in Table 1 are the fluorescence quantum yields $\left(\Phi_{\mathrm{F}}\right)$ and excited-singlet state lifetimes $\left(\tau_{\mathrm{S}}\right)$ recorded in MTHF solution at room temperature. Emission is readily detected from both chromophores and the radiative rate constants $\left(\mathrm{k}_{\mathrm{RAD}}\right)$ are comparable across the series. These latter values are in reasonable agreement with the corresponding values $\left(\mathrm{k}_{\mathrm{SB}}\right)$ calculated from the Strickler-Berg expression..$^{1}$ For all control compounds, there is good agreement between absorption and excitation spectra while fluorescence decay curves were strictly mono-exponential. Oscillator strengths (f) calculated for the first-allowed absorption transition and the corresponding transition dipole moments ${ }^{52}\left(\mu_{\mathrm{TD}}\right)$ are also given in Table 1.

The geometry around the DACH spacer moiety has no real effect on the photophysical or spectroscopic properties of these mono-chromophoric compounds (Table 1). Likewise, the presence of the spacer group does not introduce any significant electronic effects or spectroscopic perturbations. Although the amino groups will be in dynamic motion, alternating between axial and equatorial positions, this rapid exchange process does not affect the photophysical properties in any observable way. The minor variations between the various sets of compounds are well within experimental error.

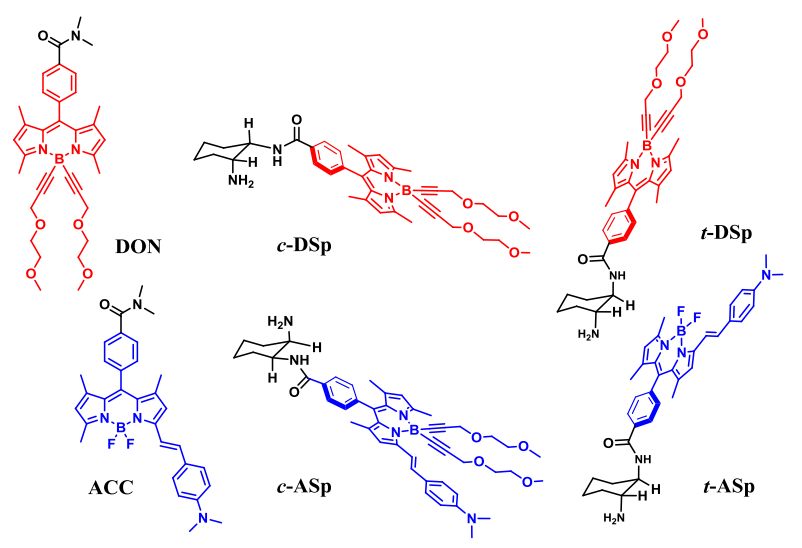

Figure 2. Representation of the various reference molecules, with and without the accompanying DACH spacer group, and their corresponding abbreviations. The donor is presented in red while the acceptor is illustrated in blue.

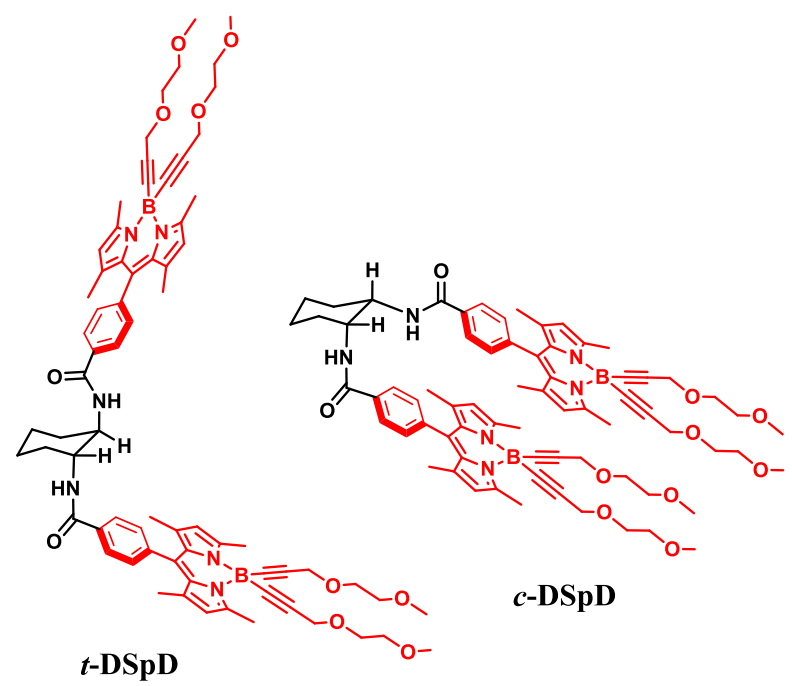

Figure 3. Molecular formulas for the symmetrical dimers proposed as additional reference compounds for the trans-geometry (seen here on the left) and the cis-geometry.

As a final control, the symmetrical dyads consisting of two identical donor units (DSpD) were studied under the same conditions (Figure 3). The derived photophysical properties are included in Table Si. Comparison with the mono-chromophoric compounds suggests that there might be some minor quenching introduced by the close proximity of the two chromophores, although there are no detectable spectral shifts. Indeed, the cis-dyad (c-DSpD) shows slightly more perturbation than the corresponding trans-dyad ( $t$-DSpD). From the measured fluorescence lifetimes, the quenching occurs with a firstorder rate constant of less than $5 \times 10^{7} \mathrm{~s}^{-1}$. It will be shown later that this value becomes insignificant in comparison to the rate constant for intramolecular EET. 


\section{Steady-state fluorescence spectroscopy with the asym- metrical dyads}

Overlap of the absorption bands for the donor and acceptor restricts selection of a wavelength region where discriminatory excitation of the donor can be realized, although this is easily achieved for the acceptor. In the case of the trans-species ( $t$-DSpA), this being a mixture of $a, a$ and $e, e$ conformations, absorption spectra of the isolated donor, acceptor, dyad and summed donor-acceptor are shown in Figure 4; the corresponding spectra for the cis-species (c-DSpA) are shown as Figure $\mathrm{S}_{14}$. From the comparison, it appears that excitation at $470 \mathrm{~nm}$ offers the best choice. There is little, if any, electronic interaction between the two chromophores across the dyad. Thus, the spacer group appears to hold apart the chromophores; this impression is supported by molecular modelling studies as will be described later. For each system, excitation at $560 \mathrm{~nm}$ leads to population of the first-allowed, singlet-excited state associated with the acceptor. Fluorescence quantum yields and excited-state lifetimes measured in MTHF are collected in Table 2 . The quantum yields derived for the dyads are reduced relative to the mono-chromophores but the lifetimes are more comparable. It is possible that the presence of the donor causes minor quenching of the acceptor fluorescence, although this does not appear to be a significant effect.

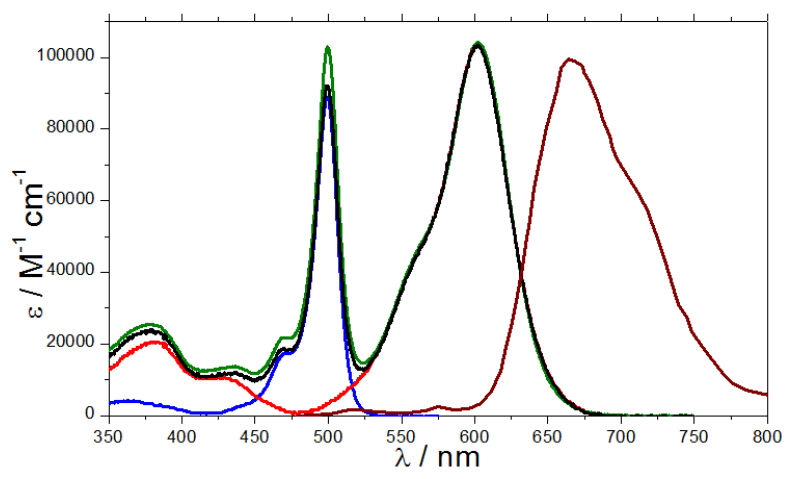

Figure 4. Absorption spectra of each component of the socalled trans-species and the fluorescence spectrum recorded for the dyad ( $t$-DSpA, brown curve) in MTHF. Individual absorption spectra refer to the donor ( $t$-DSp, blue curve), the acceptor ( $t$-SpA, red curve) and the dyad ( $t$-ASp, green curve).

In contrast, fluorescence from the donor is heavily quenched in both dyads. The preferred excitation wavelength for these measurements is $470 \mathrm{~nm}$, where the donor chromophore is responsible for $>95 \%$ of the total absorbance (Figures 4 and S2). Under these conditions, the quantum yields for donor emission are massively decreased while the lifetimes become too short $\left(\tau_{S}<50 \mathrm{ps}\right)$ to be resolved by time-correlated, single photon counting or phase modulation methods (Table 2). The extent of fluorescence quenching $\left(\mathrm{P}_{\mathrm{Q}}\right)$ exceeds $99 \%$ in each case. For the same excitation wavelength, the acceptor fluorescence is significantly increased compared to that from an equimolar mixture of donor and acceptor. Fluorescence excitation spectra are in good agreement with the absorption spectrum of the donor in the region, including the region where the donor absorbs strongly (Figures S11 and S12). These latter factors point towards electronic energy transfer (EET) as being the primary cause of extinction of donor emission for these particular dyads.

The spectral overlap integral (JDA for emission from DSp and absorption by ASp were calculated $5^{2}$ on the basis of Equation 1 and are included in Table 2. Here, the individual spectra are recorded in units of wavenumber $(v)$ and normalized to unity; $f_{\mathrm{D}}$ refers to the emission intensity for the donor and $\varepsilon_{\mathrm{A}}$ is the molar absorption coefficient for the acceptor. Again, there is no apparent effect of the spacer geometry. The derived values for JDA are relatively high, despite the observation that the respective $\mathrm{o}, \mathrm{o}$ vibrational bands do not overlap to any appreciable extent. In fact, EET from D to A is likely to populate upper vibronic modes associated with the acceptor unit. This effect is illustrated in Figure2 S15 and S16 and indicates clearly that the absorption spectrum of the acceptor exhibits a vibrational progression on the high-energy side that corresponds to a medium-frequency mode of ca. 1,170 $\mathrm{cm}^{-1}$. The excitation energies of donor and acceptor are 19,700 and 16,000 $\mathrm{cm}^{-1}$, respectively (Table 1 ). Therefore, the excess excitation energy of $3,700 \mathrm{~cm}^{-1}$ will be dissipated via this mode, which is attributed to $\mathrm{C}=\mathrm{C}$ and $\mathrm{C}=\mathrm{N}$ torsional bending modes. Direct EET will populate the third or fourth vibrational level and should be followed by fast vibrational cooling. 53

$$
\begin{aligned}
& J_{D A}=A B \int \frac{f_{D}(v)}{v^{3}} \times \frac{\varepsilon_{A}(v)}{v} d v \\
& A \int \frac{f_{D}(v)}{v^{3}} d v=B \int \frac{\varepsilon_{A}(v)}{v} d v=1 \\
& R_{C D}^{6}=\frac{\tau_{S} J_{D A}}{\hbar^{2} c}\left(\frac{s \kappa}{4 \pi \varepsilon_{0}}\left|\mu_{D}\right|\left|\mu_{A}\right|\right)^{2} \\
& S=\frac{3}{2 n^{2}+1}
\end{aligned}
$$

The Förster critical distance $\left(\mathrm{R}_{\mathrm{CD}}\right)$ for EET across these molecular dyads was calculated 54 from Equation 2 where s refers to the Onsager screening factor, $55 \mathrm{n}$ is the refractive index of the surrounding medium, $\mu$ refers to the transition dipole moment for donor or acceptor, and $\kappa$ is the orientation factor..$^{6}$ The latter term can be calculated in several ways but we opted to make use of Equation 3 where $\theta_{\mathrm{T}}$ is the angle between the transition dipole moment vectors on donor and acceptor. The corresponding angles between the molecular axis, taken as an imaginary line connecting the centers of the two transition dipole moment vectors, and the donor vector or the acceptor vector are referred to as $\theta_{D}$ or $\theta_{A}$, respectively. These angles are subject to considerable uncertainty because of certain internal rotations. We take the critical distance as being the separation between the centers of the transition dipole moment vectors for $\mathrm{D}$ and $\mathrm{A}$ at the point where the EET probability is $50 \%$. Under these specific conditions, the excited-state lifetime for the donor will be halved to $2.0 \mathrm{~ns}$. On the assumption of random orientations of the transition dipole moment vectors (i.e., $\kappa^{2}=0.67$ ), the average value for $\mathrm{R}_{\mathrm{CD}}$ is found to be around 55 A. A molecular model with the chromophores forced into the fully extended geometry corresponds to a center-to-center separation length of only 22 Å (Figure S25) such that we might anticipate very efficient EET for the asymmetrical dyads, DSpA, regardless of the geometry imposed by the spacer. 
Table 1. Compilation of the main photophysical properties recorded for the mono-chromophoric control compounds in MTHF solution at room temperature. ${ }^{a}$

\begin{tabular}{|l|l|l|l|l|l|l|}
\hline Property & DON & $t$-DSp & $c$-DSp & ACC & $t$-ASp & $c$-ASp \\
\hline$\lambda_{\text {MAX }} / \mathrm{nm}$ & 499 & 499 & 499 & 599 & 600 & 599 \\
\hline$\varepsilon_{\text {MAX }} / \mathrm{M}^{-1} \mathrm{~cm}^{-1}$ & 85,225 & 88,085 & 86,060 & 102,950 & 104,190 & 103,030 \\
\hline $\mathrm{f}$ & 0.29 & 0.28 & 0.29 & 0.65 & 0.63 & 0.60 \\
\hline$\lambda_{\text {FLU }} / \mathrm{nm}$ & 516 & 516 & 517 & 653 & 656 & 654 \\
\hline$\Phi_{\mathrm{F}}$ & 0.56 & 0.64 & 0.61 & 0.78 & 0.74 & 0.76 \\
\hline$\tau_{\mathrm{S}} / \mathrm{ns}$ & 3.8 & 4.0 & 3.9 & 4.1 & 4.0 & 4.1 \\
\hline $\mathrm{k}_{\mathrm{RAD}} / 10^{7} \mathrm{~s}^{-1}$ & 15.0 & 16.0 & 15.6 & 19.0 & 18.0 & 18.5 \\
\hline $\mathrm{k}_{\mathrm{SB}} / 10^{7} \mathrm{~s}^{-1}$ & 18.0 & 19.0 & 19.0 & 24.0 & 23.0 & 23.0 \\
\hline$\mu_{\mathrm{TD}} / \mathrm{D}$ & 2.50 & 2.43 & 2.41 & 3.21 & 3.15 & 3.20 \\
\hline $\mathrm{E}_{\text {оо }} / \mathrm{cm}^{-1}$ & 19,710 & 19,710 & 19,690 & 16,005 & 15,955 & 15,995 \\
\hline
\end{tabular}

(a) The specification of cis- or trans-geometry refers to the arrangement of the amino groups on the DACH spacer function. These can interconvert between axial and equatorial positions but not between the cis- and trans-geometries.

$$
\kappa=\cos \theta_{T}-3 \cos \theta_{D} \cos \theta_{A}
$$

\section{Time-resolved fluorescence studies with the "trans" conformer}

The measurement of EET dynamics requires better temporal resolution than is possible with time-correlated, single photon counting. This was provided 57 using up-conversion fluorescence spectroscopy. For these ultrafast measurements, excitation was provided at $515 \mathrm{~nm}$, where the donor absorbs ca. $60 \%$ of incident photons (Figure 4 ). Detection was fixed at $660 \mathrm{~nm}$, where the acceptor fluorescence is at a maximum and there is essentially no residual emission from the donor. The results are shown in Figure 5. At this wavelength, the fluorescence decay profile shows a fast component with a lifetime of ca. $15 \mathrm{ps}$, representing $40 \%$ of the initial amplitude, and a residual component having a lifetime in the nanosecond range. This latter emitting species is identified as the thermally-relaxed, excited singlet state associated with the acceptor while the 15-ps component is attributed to vibrational relaxation within the acceptor moiety. ${ }^{8}$ It is, however, the rise-times for the fluorescence signal that contain more relevant information in terms of intramolecular EET. At $66 \mathrm{onm}$, it was considered that ca. $60 \%$ of the signal should grow-in after the excitation pulse as a consequence of initial population of the donor excited state while the remaining $40 \%$ of the emission should be formed instantaneously. This latter component represents that fraction of the signal that arises from direct excitation of the acceptor. Using these restricting conditions, a relatively good fit could be obtained with a rise-time constant of 320 fs for the $60 \%$ component (Figure 5 ). This fast component is assigned to intramolecular EET across the DACH spacer.

Fluorescence from the donor was recorded and the decay profile could be analyzed to give a rough fit of $300-500$ fs. Unfortunately, the data were quite noisy and there has to be some concern over the ambiguity of assigning the decay when we have $40 \%$ direct excitation of the acceptor. For example, part of the decay curve could arise from hot emission associated

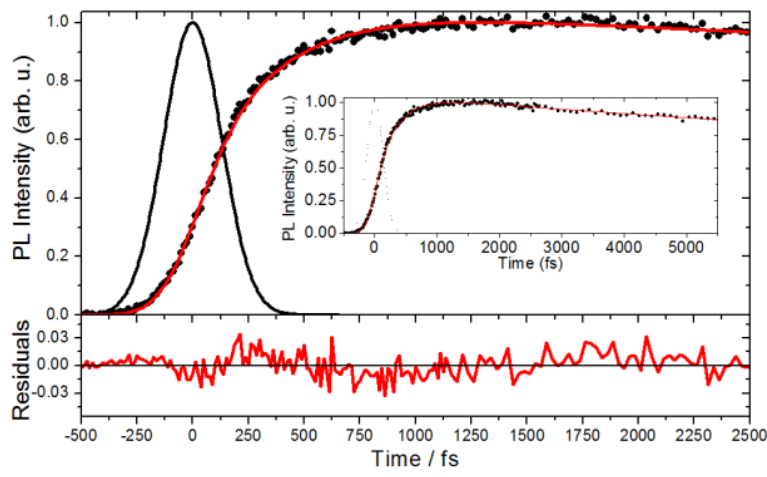

Figure 5. Example of the ultrafast time-resolved emission profile recorded following excitation of $t$-DSpA at $515 \mathrm{~nm}$, exciting $60 \%$ donor and $40 \%$ acceptor. Detection is at $660 \mathrm{~nm}$, being solely due to emission from the acceptor. The rise of the emission is best fitted with a time constant of $320 \mathrm{fs}$, constituting $60 \%$ of the rise (fixed), the other $40 \%$ being instantaneous. A decay is observed on longer timescales (inset), fitted to $\sim 15$ ps, with a further long-lived offset. The dotted line is the instrument response function and the bottom panel shows the weighted residuals.

with the acceptor moiety. Thus, under these conditions, it is not entirely certain that the process being monitored is related solely to electronic energy transfer from donor to acceptor.

In principle, further information 59 about the EET process might be sought from time-resolved depolarization of the fluorescence signal at $660 \mathrm{~nm}$, as shown in Figure 6. This type of analysis is severely hindered, however, by the fact that some $40 \%$ of the acceptor-based fluorescence is formed by direct excitation. This direct population of the emitting state will most likely alter the observed anisotropy values. It is clear, however, that intramolecular EET causes a large change in anisotropy, indicating a significant change in emission dipole angle, ${ }^{60}$ as the final measured value is close to -0.1. Since direct excitation 
is expected to give a positive, but unknown, anisotropy value, it is reasonable to suppose that the change in transition dipole accompanying EET is likely to be close to $90^{\circ}$ (see later).

Table 2. Summary of spectroscopic properties recorded for the asymmetrical dyads in MTHF solution.

\begin{tabular}{|c|c|c|}
\hline Property & $t$-DSpA & $c$-DSpA \\
\hline$\lambda_{\mathrm{ABS}} / \mathrm{nm}$ & $499^{(\mathrm{a})}: 602^{(\mathrm{b})}$ & $499^{(a)}: 603^{(b)}$ \\
\hline$\varepsilon_{\text {MAX }} / \mathrm{M}^{-1} \mathrm{~cm}^{-1}$ & $\begin{array}{l}103,040^{(\mathrm{a})}: \\
102,385^{(\mathrm{b})}\end{array}$ & $\begin{array}{l}102,250^{(a)}: \\
100,000^{(b)}\end{array}$ \\
\hline$\lambda_{\mathrm{FLU}} / \mathrm{nm}$ & $516^{(\mathrm{a})}: 664^{(\mathrm{b})}$ & $524^{(\mathrm{a})}: 669^{(\mathrm{b})}$ \\
\hline$\Phi_{\mathrm{F}}(\mathrm{c})$ & 0.42 & 0.45 \\
\hline$\tau_{\mathrm{S}} / \mathrm{ns}$ & $3.6^{(b)}$ & $3 \cdot 5^{(b)}$ \\
\hline $\mathrm{P}_{\mathrm{Q}} / \%{ }^{(\mathrm{d})}$ & 99.8 & 99.2 \\
\hline $\mathrm{J}_{\mathrm{DA}} / \mathrm{cm}$ & 0.0034 & 0.0041 \\
\hline $\mathrm{R}_{\mathrm{CD}} / \AA$ & 53.5 & $55 \cdot 9$ \\
\hline
\end{tabular}

(a) Refers to the donor moiety. (b) Refers to the acceptor moiety. (c) Quantum yield for the acceptor following excitation into the donor at $470 \mathrm{~nm}$. (d) Calculated by comparison of the donor fluorescence yield with that of the corresponding symmetrical dyad with excitation at $470 \mathrm{~nm}$.

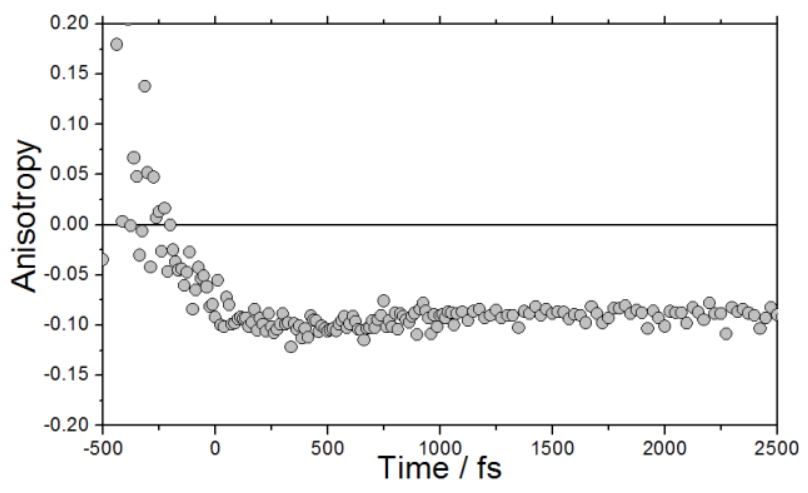

Figure 6. Ultrafast fluorescence anisotropy of $t$-DSpA following excitation at $515 \mathrm{~nm}$ and with detection at $660 \mathrm{~nm}$. Anisotropy begins positive before turning negative, reaching a minimum value of ca. -o.1.

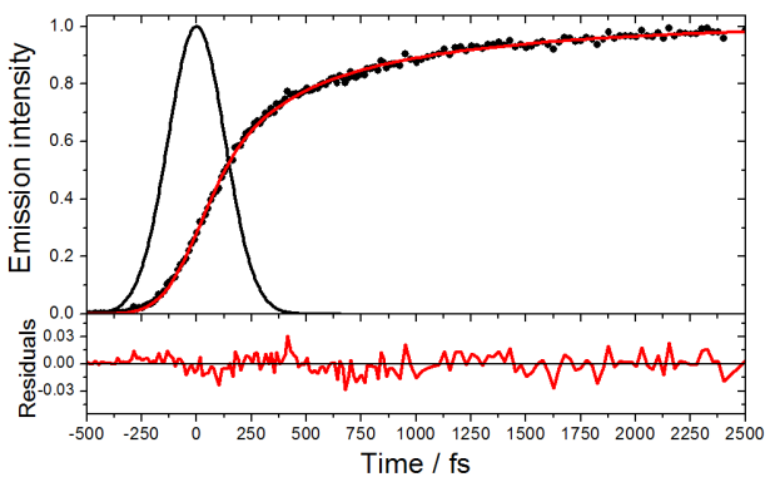

Figure 7. Example of time-resolved emission detected at 660 $\mathrm{nm}$ following excitation of $c$-DSpA at $515 \mathrm{~nm}$. The rise-time amplitude of $60 \%$ is split into two $30 \%$ components (fixed) with the time constants given in the text. Good agreement with the experimental data is obtained, as evidenced by the relatively minor features in the residuals shown at the bottom.

\section{Time-resolved fluorescence studies with the "cis" con- former}

The only difference between the cis- and trans-dyads relates to the geometry around the central spacer unit. Similar measurements to those described above for the trans-conformer were made for the cis-conformer (Figure 7). The relevant absorption spectra (Figure S2) were analyzed to confirm that excitation at $515 \mathrm{~nm}$ will lead to ca. $40 \%$ direct excitation of the acceptor moiety. In the case of $c$-DSpA, there were no obvious signs 53,58 of vibrational cooling for the emission signal monitored at $660 \mathrm{~nm}$. Instead, the emission was observed to decay with a nanosecond time constant, as already described. In this case, the rise time could not be accounted for as a combination of an instantaneous growth, with an amplitude of ca. $40 \%$, and a slower component (Figure S18-S22). Satisfactory fits could be obtained with the rise-time appearing as the sum of two exponential components, contributing a total of $60 \%$ towards the amplitude, and an instantaneous appearance of ca. 40\% amplitude (Figure 7). The fitted rise-time constants were found to be 250 and 970 fs. As above, these fast components are attributed to intramolecular EET from donor to acceptor. One possibility for the occurrence of two lifetimes is that the dyad exists in two conformations that do not interconvert on the relevant time scale (see below). Fluorescence anisotropy measurements confirm that there must be a substantial change in the angle between the relevant transition dipoles during the EET (Figure $\mathrm{S}_{17}$ ).

\section{Molecular modelling studies with the asymmetrical dy- ads}

The ultrafast fluorescence measurements indicate rapid intramolecular EET in both asymmetrical dyads, despite the modest spectral overlap integrals. Similar timescales are indicated for the two structures but it is suggested that the cisconformer co-exists in the form of two structural groups that do not equilibrate on the timescale for EET. Both cis- and trans-isomers are anticipated to undergo fast equilibration between axial and equatorial species ${ }^{61}$ but such exchange is unlikely to compete with EET across the spacer group. There are several possible sites for internal rotation and the amide bonds can themselves alternate between cis- and trans-geometries. ${ }^{62}$ This adds up to a complex and dynamic topological picture. To help restrict this structural landscape, a series of molecular modelling studies was undertaken with each dyad dispersed in a solvent reservoir having a dielectric constant of 10. Starting structures were generated to correspond to the generic picture portrayed in Chart 1.

We begin by consideration of the preferred molecular topology for the cis-conformer c-DSpA, which requires one chromophore to be in an axial configuration and the second chromophore to be located at an equatorial site (chart 1). The energy-minimized structures demanded an equatorial donor and an axial acceptor; we can specify this species as being $c$ $\mathrm{D}_{\mathrm{E}} \mathrm{SpA}_{\mathrm{A}}$. Such species were generally ca. $10 \mathrm{~kJ} / \mathrm{mol}$ more stable than the corresponding $c-\mathrm{D}_{\mathrm{A}} \mathrm{Sp}_{\mathrm{E}}$ species, possibly reflecting the larger size of the acceptor unit. In order to simplify the situation, we ignore the less stable family of conformers and restrict attention to $c-\mathrm{D}_{\mathrm{E}} S \mathrm{PA} \mathrm{A}_{\mathrm{A}}$. Here, the lowest energy conformation has both amide groups held in the trans-geometry. 
This leads to a relatively closed structure with a donor-acceptor center-to-center separation $\left(\mathrm{R}_{\mathrm{DA}}\right)$ of ca. $13.6 \AA$. Partial rotation around the $\mathrm{C}-\mathrm{C}$ connecting bond between the carbonyl group and the meso-phenyl ring is possible for both donor and acceptor and amplifies the variation in mutual orientations but without really affecting the separation.

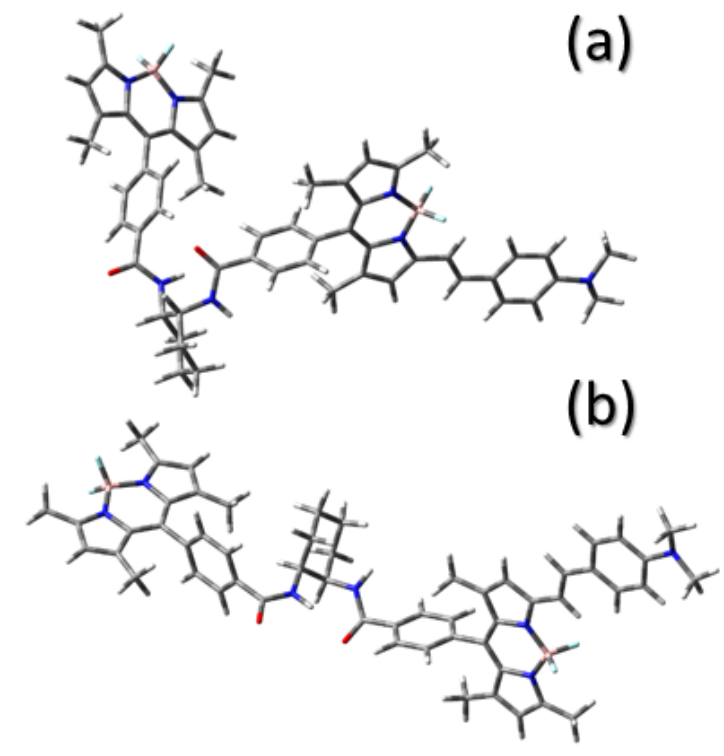

Figure 8. The two lowest-energy structures computed (PBEo/6-311G(d)/PCM) for $c-\mathrm{D}_{\mathrm{E}} \mathrm{SpA}_{\mathrm{A}}$. (a) Both amide groups are in the trans-geometry (i.e., head-to-tail configuration), giving rise to a relatively closed structure. (b) For this open form, the donor amide is in the cis-geometry but the acceptor amide has the trans-geometry.

A second conformer exists that has the donor amide group in the cis-geometry but retains a trans-geometry for the amide linker adjacent to the acceptor unit. This conformer is partially stabilized by a hydrogen bond (H-O distance of $2.7 \AA$ ) between the two amide residues but has a relatively open structure with a center-to-center separation of ca. 17.7 $\AA$. The computed energy difference between these two structures is less than $4 \mathrm{~kJ} / \mathrm{mol}$ in favor of the closed form under these conditions. Interestingly, the cis-amide becomes more favorable in less polar solvents, in part because of the hydrogen bond. Again, partial rotation around the $\mathrm{C}-\mathrm{C}$ connection increases the variety of possible orientations but does not greatly alter the separation distance. It is not possible, because of severe steric crowding, for the acceptor amide group to isomerize to or exist in the cis-geometry.

The two lowest-energy conformations are illustrated by Figure 8 and a description of all the calculated structures is given in the Supporting Information. For $c-\mathrm{D}_{\mathrm{E}} \mathrm{SpA}_{\mathrm{A}}$, isomerization of the donor amide linkage involves a rotational energy barrier of ca. $95 \mathrm{~kJ} / \mathrm{mol}$. A point of interest concerns the apparent identification of two preferred conformations that differ substantially with respect to mutual alignment of the donor and acceptor units. The energy difference between these two species is small but sensitive to the polarity of the solvent. This conformational balance could, in principle, account for the time-resolved emission results. It might be noted that FTIR spectra confirm the involvement of an intramolecular hydrogen bond.
We turn attention now to $t$-DSpA, where both donor and acceptor functions are expected to be either axial or equatorial and where, according to Chart 1, these residues will appear on opposite sides of the spacer group. The calculations indicate that the species with both donor and acceptor held at axial sites, $t-\mathrm{D}_{\mathrm{A}} \mathrm{SpA}_{\mathrm{A}}$, is more stable by ca. $15 \mathrm{~kJ} / \mathrm{mol}$ than the corresponding equatorial conformer, $t-\mathrm{D}_{\mathrm{E}} \mathrm{SpA}_{\mathrm{E}}$. To simplify matters, we eliminate this latter family of structures from further consideration. Furthermore, it is not possible to arrange for both amide groups to adopt the cis-configuration because this structure requires the two chromophores to occupy the same space. In contrast, two structures could be identified with one amide having the cis-configuration and the second amide being in the trans-configuration (Figure 9). These two energyminimized geometries are essentially isoenergetic and are therefore likely to co-exist in solution at ambient temperature. The two computed structures are relatively closed but cannot be interconverted under normal conditions. This is because the computed rotational barriers for the amide bonds are far too high; individual barriers being $83 \mathrm{~kJ} / \mathrm{mol}$ for the donor amide and $81 \mathrm{~kJ} / \mathrm{mol}$ for the acceptor amide. Internal hydrogen bonding is not possible for $t-\mathrm{D}_{\mathrm{A}} \mathrm{SpA}_{\mathrm{A}}$. (a)

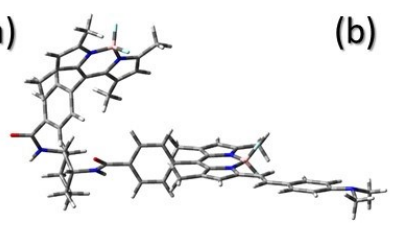

(c)

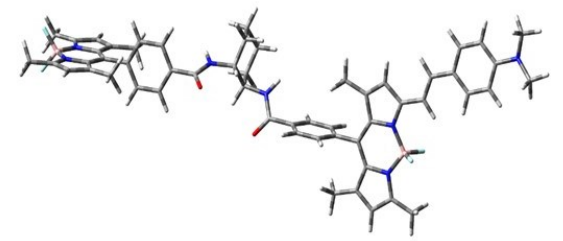

Figure 9. Stable conformations (PBEo/6-311G(d)/PCM) for $t$ $\mathrm{D}_{\mathrm{A}} \mathrm{SpA}_{\mathrm{A}}$; (a) donor amide has the cis-geometry but the acceptor amide is in the trans-configuration, (b) donor amide has the trans-geometry but the acceptor amide is in the cis-configuration, and (c) both amides are in the trans-geometry.

These calculations indicate that the structure having both amide groups in the trans-configuration is somewhat less stable than the mixed trans/cis species mentioned above by ca. 5 $\mathrm{kJ} / \mathrm{mol}$. This energy difference increases in less polar solvents. The reactants are now well separated, with a center-to-center separation distance of ca. 20 Å (Figure 9). Restricted rotation around the meso-phenyl linkages affects the molecular orientation but not the separation distance. For this species, full isomerization of the amide groups in unlikely to occur in solution since the computed rotational barriers are ca. 80 $\mathrm{kJ} / \mathrm{mol}$. Without being too specific, this open conformation is not conducive to fast EET because of the large separation ${ }^{6}$ and unfavorable orientation. ${ }^{64}$ This situation is in contrast to that of the mixed trans/cis conformers where the geometry appears more consistent with rapid intramolecular EET. We might anticipate, on the basis of these two families of conformers, to observe dual-exponential kinetics for growth of the excited-state localized on the acceptor, in addition to the 
instantaneous growth due to direct excitation. Such kinetics are observed for $c$-DSpA but not for $t$-DSpA. This points to an apparent inconsistency between the time-resolved emission studies and the molecular modelling.

The molecular modelling studies, however, do not reflect the relatively high dipole moment of the acceptor unit and its interaction with the surrounding solvent. It has already been shown ${ }^{65,66}$ that the photophysical properties of the acceptor depend markedly on the polarity of the solvent. This species is stabilized by polar solvents. Within this context, it is possible to rationalize the apparent discrepancy between the two studies: To do this, we invoke the idea of a solvatophobic effect $^{67}$ in which polar solvents, such as acetonitrile, stabilize the acceptor unit without affecting the donor. In less polar solvents, such as tetrahydrofuran, the acceptor seeks to minimize contact with the solvent by moving towards the closed conformations. Within this premise, $t-\mathrm{D}_{\mathrm{A}} \mathrm{SpA}_{\mathrm{A}}$ favors the mixed trans-cis conformers shown in Figure 9 and, as such, is expected to exhibit rapid intramolecular EET. It might be mentioned that similar solvatophobic effects have been reported $^{68,69}$ for related BODIPY-based compounds where largescale structural changes have been observed on changing the polarity of the solvent. The same behavior should hold for $c-D_{\mathrm{E}} \mathrm{SpA}_{\mathrm{A}}$ except that the internal hydrogen bond between the two amide linkages helps to stabilize the open conformation (Figure 8). Of course, hydrogen bonding is well known to stabilize the structures of peptides ${ }^{70}$ and proteins ${ }^{71}$ but is less common in synthetic dyads. ${ }^{72}$ The involvement of an O...H-N bond, unique to the open form of $c-\mathrm{D}_{\mathrm{E}} \mathrm{SpA}_{\mathrm{A}}$, brings a simple explanation to the observed kinetic behavior.

\section{Comparison with Förster dipole-dipole theory}

The structural parameters determined by molecular modelling, together with the spectroscopic data, were used to compute the rates of EET across the DACH spacer unit. The theory ${ }^{1,73}$ assumes through-space EET between reactants that are not subject to mutual electronic interactions. Thus, Equation 4 was used to calculate the rate constant $\left(\mathrm{k}_{\mathrm{EET}}\right)$ for intramolecular EET in each of the recognized conformers on the assumption that the electronic coupling matrix element $\left(V_{D A}\right)$ refers solely to Coulombic interactions. In the first instance, the distance parameter was taken as the separation between the centers of the respective transition dipole moment vectors associated with donor and acceptor (see Supporting Information for a listing of these values). The average separation distances $\left(R_{D A}\right)$ are comparable to the sum of the transition dipole moment vectors taken from the molecular models and there has to be serious concerns about the validity of using Förster theory under such circumstances. ${ }^{74-76}$ Other concerns include the exact form of the solvent screening factor 77 (s), the involvement of multipole interactions at short separations, 78 and the uncertainty about the calculated orientation factor ${ }^{79}$ (к).

$$
\begin{aligned}
& k_{E E T}=\frac{\left|V_{D A}\right|^{2}}{\hbar^{2} c} J_{D A} \\
& V_{D A}=\frac{s \kappa}{4 \pi \varepsilon_{0}} \frac{\left|\mu_{D}\right|\left|\mu_{A}\right|}{R_{C C}^{3}}
\end{aligned}
$$

On persisting with Equation 4, we find that very fast through-space EET is expected for the mixed trans-cis amide conformers projected for $t-\mathrm{D}_{\mathrm{A}} \mathrm{SpA}_{\mathrm{A}}$. Individual values are $1.9 \mathrm{x}$ $10^{12} \mathrm{~s}^{-1}\left(\mathrm{R}_{\mathrm{DA}}=7.8 \AA\right)$ for the cis-amide at the acceptor site and $1.8 \times 10^{12} \mathrm{~s}^{-1}\left(\mathrm{R}_{\mathrm{DA}}=12.7 \AA\right)$ with the cis-amide on the donor side. The average value is surprisingly close to the experimental

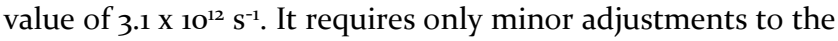
geometry to bring the experimental and calculated $k_{\mathrm{EET}}$ values into perfect agreement and it should be stressed that there are large errors associated with the calculated orientation factors. ${ }^{80}$ That the rates for the two proposed conformers are closely comparable is in line with the observation of a single kinetic term derived from the time-resolved emission studies. In contrast, the rate constant calculated for the open confor-

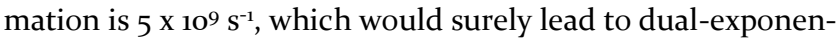
tial kinetics for the EET event. Allowing for higher-order contributions to the coupling element, such as quadrupole-dipole interactions, ${ }^{81}$ will increase the calculated $\mathrm{k}_{\mathrm{EET}}$ values for the mixed cis-trans species. The same is true if due allowance is made for the nearby polar acceptor inducing a partial dipole for the nonpolar donor. ${ }^{82}$

Similar calculations were made for $c-D_{E} S p A_{A}$. Thus, the calculated rate constant for intramolecular EET for the species with both amides held in the trans-configuration $\left(\mathrm{R}_{\mathrm{DA}}=13.7\right.$ $\AA$ ) is $2.0 \times 10^{12} \mathrm{~s}^{-1}$. This value agrees well with the experimental rate constant of $4 \times 10^{12} \mathrm{~s}^{-1}$. The second species, having the donor amide in the cis-geometry and the acceptor amide held in the trans-arrangement $\left(\mathrm{R}_{\mathrm{DA}}=13.8 \AA\right.$ ) has a calculated $\mathrm{k}_{\mathrm{EET}}$ of

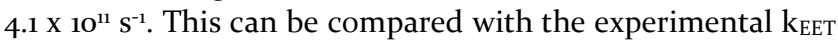
of $1 \times 10^{12} \mathrm{~s}^{-1}$. The calculations consistently underestimate the experimental values by a factor of ca. 2 -fold but, considering the uncertainties and doubts involved in applying the ideal dipole approximation to non-spherical reagents at short separations, this level of agreement is astonishing. At the least, it is possible to assign the two $\mathrm{k}_{\mathrm{EET}}$ values derived for $c-\mathrm{D}_{\mathrm{E}} \mathrm{Sp} \mathrm{A}_{\mathrm{A}}$ to particular structures.

For both systems, replacing the ideal dipole approximation with the extended dipole approached introduced by $\mathrm{Kuhn}^{83}$ leads to similar conclusions to those reached above. The proposed molecular geometries give rise to computed coupling elements that can be used to give $\mathrm{k}_{\mathrm{EET}}$ values in quite good agreement with the experimental results. The advantage of the extended dipole moment is that it avoids the reliance on mutual angles but requires information about the dipole lengths (Figures S25 and S26). These are not so easy to establish for the acceptor. Nonetheless, these findings are further testimony that the projected molecular structures allow a good understanding of the rates of very fast through-space EET in these system.

\section{CONCLUSIONS}

This work describes intramolecular electronic energy transfer within a molecular dyad where the reactants are held in relatively close proximity and presumably in dynamic equilibrium between different conformations. The spacer group is not expected to be effective at promoting through-bond electronic interactions and instead it is anticipated that EET will take place by way of long-range through-space interactions. The application of ultrafast fluorescence spectroscopy has confirmed highly effective EET from donor to acceptor and has allowed determination of the corresponding rates of intramolecular EET. The derived rates are extremely fast (i.e., subps) but, because we monitor growth of the excited state resident on the acceptor moiety, these can be safely attributed to intramolecular EET. The results are fully consistent with one dyad existing as a distribution of conformers with similar topologies but the other dyad showing two groups of conformations that do not equilibrate on the relevant timescale. This 
disparity is explained in terms of an internal hydrogen bond while the molecular shape is believed to be controlled, in part, by solvatophobic effects associated with the polar acceptor. If correct, it is remarkable that a single hydrogen bond can influence the 3 -dimensional structure of a large molecule having multiple sites where topological ambiguity can exist. The possibility idea that an intramolecular hydrogen bond could overcome the solvatophobic effect and control the geometry of a large multi-component molecule seems worthy of more detailed examination.

The DACH scaffold has not been used previously as a spacer for molecular dyads but could be a valuable building block for constructing chiral architectures. The amide connections invariably introduce structural complexities but these might be controlled by provision of hydrogen bonding sites. For the non-spherical dyads studied here, the rates of through-space EET are consistent with the results of calculations $^{1}$ made with the ideal dipole approximation. The level of agreement between experiment and theory is surprising, especially given the very high rates of EET. Förster theory consistently underestimates the rates but only by a factor of about 2 -fold. It will now be challenging to design new molecular dyads for through-space EET across shorter distances than those involved here. Indeed, for $t-\mathrm{D}_{\mathrm{A}} \mathrm{SpA}_{\mathrm{A}}$, the shortest center-tocenter separation is only $7.8 \AA$ which is comparable to that for certain natural light-harvesting antennas where distances fall within the range 5-20 $\AA .84$ Despite the close proximity, there are no obvious signs of interchromophore coupling in these dyads, at least in liquid phase. As such the scaffold could be a useful medium for promoting singlet exciton fission ${ }^{85}$ where two identical chromophores should be held closely together. ${ }^{86}$ It will also be instructive to evaluate the performance of the dyads in thin films.

\section{ASSOCIATED CONTENT}

Supporting Information includes experimental details, compound characterization, additional spectroscopic and structural information, and parameters used for Förster calculations. This material is available free of charge via the Internet at http://pubs.acs.org.

\section{AUTHOR INFORMATION}

\section{Corresponding Author}

*Anthony Harriman: ORCID oooo-0oo3-0679-2232. e-mail: anthony.harriman@ncl.ac.uk. tel: +44 191208 866o.

\section{Present Addresses}

†PS: Department of Physics, Durham University, South Road, Durham, DH1 3LE, United Kingdom. GJH: WestCHEM, School of Chemistry, University of Glasgow, Joseph Black Building, University Avenue, Glasgow, G12 8QQ, United Kingdom.

\section{Author Contributions}

All authors have given approval to the final version of the manuscript.

\section{ACKNOWLEDGMENT}

We thank Newcastle University for financial support of this work. We acknowledge the contributions made by Alexandra Sutter in establishing the synthetic procedures used here.

\section{ABBREVIATIONS}

EET electronic energy transfer; DACH diaminocyclohexyl; TSTU O-(N-succinimidyl)-N,N,N',N'-tetramethyjuronium tetrafluoroborate; NMR nuclear magnetic resonance.

\section{REFERENCES}

(1) Förster, T. Intermolecular Energy Migration and Fluorescence. Ann. Phys. 1948, 2, 55-75.

(2) Garcia-Parajo, M. F.; Hernando, J.; Mosteiro, G. S.; Hoogenboom, J. P.; van Dijk, E. M. H. P.; van Hulst, N. F. Energy Transfer in Single-Molecule Photonic Wires. ChemPhysChem 2005, 6, 819827.

(3) Fan, J.; Hu, M.; Zhan, P.; Peng, X. Energy Transfer Cassettes Based on Organic Fluorophores: Construction and Applications in Ratiometric Sensing. Chem. Soc. Rev. 2013, 42, 29-43.

(4) Jares-Erijman, E. A.; Jovin, T. M. FRET Imaging. Nat. Biotechnol. 2003, 21, 1387-1395.

(5) Suresh, M.; Mandal, A. K.; Kesharwani, M. K.; Adarsh, N. N.; Ganguly, B.; Kanaparthi, R. K.; Samanta, A.; Das, A. Folding and Unfolding Movements in a [2] Pseudorotaxane. J. Org. Chem. 2011, $76,138-144$.

(6) Holden, S. J.; Uphoff, S.; Hohlbein, J.; Yadin, D.; Le Reste, L.; Britton, O. J.; Kapanidis, A. N. Defining the Limits of SingleMolecule FRET Resolution in TIRF Microscopy. Biophys. J. 2010, 99, 3102-3111.

(7) Sahoo, H.; Roccatano, D.; Hennig, A.; Nau, W. M. A 1o-Angstrom Spectroscopic Ruler Applied to Short Polyprolines. J. Am. Chem. Soc. 2007, 129, 9762-9772.

(8) Chen, Y.; O'Donoghue, M. B.; Huang, Y. F.; Kang, H. Z.; Phillips, J. A.; Chen, X. L.; Estevez, M. C.; Yang, C. Y. J.; Tan, W. H. A Surface Energy Transfer Nanoruler for Measuring Binding Site Distances on Live Cell Surfaces. J. Am. Chem. Soc. 2010, 132, 1655916570 .

(9) Sindbert, S.; Kalinin, S.; Hien, N.; Kienzler, A.; Clima, L.; Bannwarth, W.; Appel, B.; Muller, S.; Seidel, C. A. M. Accurate Distance Determination of Nucleic Acids via Förster Resonance Energy Transfer: Implications of Dye Linker Length and Rigidity. J. Am. Chem. Soc. 2011, 133, 2463-2480.

(10) Zhang, J.; Fu, Y.; Lakowicz, J. R. Enhanced Förster Resonance Energy Transfer (FRET) on a Single Metal Particle. J. Phys. Chem. C 2007, 111, 50-56.

(11) Takakusa, H.; Kikuchi, K.; Urano, Y.; Kojima, H.; Nagano, T. A Novel Design Method of Ratiometric Fluorescent Probes Based on Fluorescence Resonance Energy Transfer Switching by Spectral Overlap Integral. Chem. Eur. J. 2003, 9, 1479-1485.

(12) Deniz, A. A.; Dahan, M.; Grunwell, J. R.; Ha, T. J.; Faulhaber, A. E.; Chemla, D. S.; Weiss, S.; Schultz, P. G. Single-Pair Fluorescence Resonance Energy Transfer on Freely Diffusing Molecules: Observation of Förster Distance Dependence and Subpopulations. Proc. Natl. Acad. Sci. USA 1999, 96, 3670-3675.

(13) Ha, T.; Enderle, T; Ogletree, D. F.; Chemla, D. S.; Selvin, P. R.; Weiss, S. Probing the Interaction between Two Single Molecules: Fluorescence Resonance Energy Transfer between a Single Donor and a Single Acceptor. Proc. Natl. Acad. Sci. USA 1996, 93, 6264-6268.

(14) Rogach, A. L.; Klar, T. A.; Lupton, J. M.; Meijerink, A.; Feldmann, J. Energy Transfer with Semiconductor Nanocrystals. J. Mater. Chem. 2009, 19, 1208-1221.

(15) Alamiry, M. A. H.; Hagon, J. P.; Harriman, A.; Bura, T.; Ziessel, R. Resolving the Contribution due to Förster-Type Intramolecular Electronic Energy Transfer in Closely Coupled Molecular Dyads. Chem. Sci. 2012, 3, 1041-4048.

(16) Langhals, H.; Esterbauer, A. J.; Walter, A.; Riedle, E.; Pugliesi, I. Förster Resonant Energy Transfer in Orthogonally Arranged Chromophores. J. Am. Chem. Soc. 2o10, 132, 16777-16782. 
(17) Bothner-By, A. A.; Dadok, J.; Johnson, T. E.; Lindsey, J. S. Molecular Dynamics of Covalently-Linked Multi-Porphyrin Arrays. J. Phys. Chem. 1996, 100, 17551-17557.

(18) Semiao, F. L.; Furuya, K.; Milburn, G. J. Vibration-Enhanced Quantum Transport. New J. Phys. 2010, 12, 083033.

(19) Bredas, J. L.; Sargent, E. H.; Scholes, G. D. Photovoltaic Concepts Inspired by Coherence Effects in Photosynthetic Systems. Nat. Mater. 2017, 16, 35-44.

(20) Hagler, T. W.; Pakbaz, K.; Heeger, A. J. Polarized Electroabsorption Spectroscopy of a Soluble Derivative of Poly(p-phenylenevinylene) Oriented by Gel Processing in Polyethylene - Polarization Anisotropy, the Off-Axis Dipole Moment and Excited State Delocalization. Phys. Rev. B 1994, 49, 10968-10975.

(21) Czikklely, V.; Forsterling, H. D.; Kuhn, H. Extended Dipole Model for Aggregates of Dye Molecules. Chem. Phys. Lett. 1970, 6, 207-210.

(22) Harriman, A.; Mallon, L.; Ziessel, R. Energy Flow in a Purpose-Built Cascade Molecule Bearing Three Distinct Chromophores Attached to the Terminal Acceptor. Chem. Eur. J. 2008, 14, 11461-11473.

(23) Evans, C. E.; Song, Q.; Bohn, P. W.; Influence of Molecular Orientation and Proximity on Spectroscopic Line-Shape in Organic Monolayers. J. Phys. Chem. 1993, 97, 12302-12308.

(24) Beenken, W. J. D.; Pullerits, T. Excitonic Coupling in Polythiophenes: Comparison of Different Calculation Methods. J. Chem. Phys. 2004, 120, 2490-2495.

(25) Munoz-Losa, A.; Curutchet, C.; Krueger, B. P.; Hartsell, L. R.; Mennucci, B. Fretting About FRET: Failure of the Ideal Dipole Approximation. Biophys. J. 2009, 96, 4779-4788.

(26) Ziessel, R.; Alamiry, M. A. H; Elliott, K. J.; Harriman, A. Exploring the Limits of Förster Theory for Energy Transfer at a Separation of 20 Angstrom. Angew. Chem., Int. Ed. 2009, 48, 27722776.

(27) Yeow, E. K. L.; Ghiggino, K. P.; Reek, J. N. H.; Crossley, M. J.; Bosman, A. W.; Schenning, A. P. H. J.; Meijer, E. W. The Dynamics of Electronic Energy Transfer in Novel Multiporphyrin Functionalized Dendrimers: A Time-Resolved Fluorescence Anisotropy Study. J. Phys. Chem. B 200o, 104, 2596-26o6.

(28) Varnavski, O. P.; Ostrowski, J. C.; Sukhomlinova, L.; Twieg, R. J.; Bazan, G. C.; Goodson, T. Coherent Effects in Energy Transport in Model Dendritic Structures Investigated by Ultrafast Fluorescence Anisotropy Spectroscopy. J. Am. Chem. Soc. 2002, 124, 1736-1743.

(29) Paulo, P. M. R.; Gronheid, R.; De Schryver, F. C.; da Costa, S. M. B. Porphyrin-Dendrimer Assemblies Studied by Electronic Absorption Spectra and Time-Resolved Fluorescence. Macromolecules 2003, 36, 9135-9144.

(30) Nakamura, Y.; Aratani, N.; Osuka, A. Cyclic Porphyrin Arrays as Artificial Photosynthetic Antenna: Synthesis and Excitation Energy Transfer. Chem. Soc. Rev. 2007, 36, 831-845.

(31) Head, N. J.; Oliver, A. M.; Look, K.; Lokan, N. R.; Jones, G. A.; Paddon-Row, M. N. Novel U-Shaped Systems Containing an Imide-Functionalized Cleft for the Study of Solvent-Mediated Electron Transfer and Energy Transfer: Synthesis and Binding Studies. Angew. Chem., Int. Ed. 1999, 38, 3219-3222.

(32) Meineke, D. N. H.; Bossi, M. L.; Ta, H.; Belov, V. N.; Hell, S. W. Bichromophoric Compounds with Orthogonally and Parallelly Arranged Chromophores Separated by Rigid Spacers. Chem. Eur. J. 2017, 23, 2469-2475.

(33) Hablot, D.; Ziessel, R.; Alamiry, M. A. H.; Bahraidah, E.; Harriman, A. Nanomechanical Properties of Molecular-Scale Bridges as Visualised by Intramolecular Electronic Energy Transfer. Chem. Sci. 2013, 4, 444-453.

(34) Harriman, A.; Alamiry, M. A. H.; Hagon, J. P.; Hablot, D.; Ziessel, R. Through-Space Electronic Energy Transfer Across
Proximal Molecular Dyads. Angew. Chem., Int. Ed. 2013, 52, 66116615.

(35) van Hal, P. A.; Beckers, E. H. A.; Meskers, S. C. J.; Janssen, R. A. J.; Jousselme, B.; Blanchard, P.; Roncali, J. Orientational Effect on the Photophysical Properties of Quaterthiophene-C-6o Dyads. Chem. Eur. J. 2002, 8, 5415-5429.

(36) Schwartz, B. J. Conjugated Polymers as Molecular Materials: How Chain Conformation and Film Morphology Influence Energy Transfer and Interchain Interactions. Ann. Rev. Phys. Chem. 2003, 54, 141-172.

(37) Karam, P.; Ngo, A. T.; Rouiller, I.; Cosa, G. Unravelling Electronic Energy Transfer in Single Conjugated Polyelectrolytes Encapsulated in Lipid Vesicles. Proc. Natl. Acad. Sci. USA 2010, 107, 17480-17485.

(38) Lv, W.; Li, N.; Li, Y. L.; Li, Y.; Xia, A. D. Shape-Specific Detection Based on Fluorescence Resonance Energy Transfer Using a Flexible Water-Soluble Conjugated Polymer. J. Am. Chem. Soc. 2006, 128, 10281-10287.

(39) Ulrich, G.; Ziessel, R.; Harriman, A. The Chemistry of Fluorescent Bodipy Dyes: Versatility Unsurpassed. Angew. Chem., Int. Ed. 2008, 47, 1184-1201.

(40) Bennani, Y. L.; Hanessian, S. Trans-1,2-Diaminocyclohexane Derivatives as Chiral Reagents, Scaffolds and Ligands for Catalysis: Applications in Asymmetric Synthesis and Molecular Recognition. Chem. Rev. 1997, 97, 3161-3196.

(41) Gasparrini, F.; Lunazzi, L.; Misiti, D.; Villani, C. Organic Stereochemistry and Conformational Analysis from Enantioselective Chromatography and Dynamic Nuclear Magnetic Resonance Measurements. Acc. Chem. Res. 1995, 28, 163-170.

(42) Santoso, Y.; Torella, J. P.; Kapanidis, A. N. Characterizing Single-Molecule FRET Dynamics with Probability Distribution Analysis. ChemPhysChem 2010, 11, 2209-2219.

(43) Goze, C.; Ulrich, G.; Ziessel, R. Tetrahedral Boron Chemistry for the Preparation of Highly Efficient "Cascatelle" Devices. J. Org. Chem. 2007, 72, 313-322.

(44) Thakare, S.; Stachelek, P.; Mula, S.; More, A. B.; Chattopadhyay, S.; Ray, A. K.; Sekar, N.; Ziessel, R.; Harriman, A. SolventDriven Conformational Exchange for Amide-Linked Bichromophoric BODIPY Derivatives. Chem. Eur. J. 2o16, 22, 14356-14366.

(45) Baruah, M.; Qin, W. W.; Flors, C.; Hofkens, J.; Vallee, R. A. L.; Beljonne, D.; Van der Auweraer, M.; De Borggraeve, W. M.; Boens, N. Solvent and pH Dependent Fluorescent Properties of a Dimethylaminostyryl Boron Dipyrromethene Dye in Solution. J. Phys. Chem. A 2006, 110, 5998-6009.

(46) Ziessel, R.; Harriman, A. Artificial Light-Harvesting Antennae: Electronic Energy Transfer by Way of Molecular Funnels. Chem. Comm. 2011, 47, 611-631.

(47) Alamiry, M. A. H.; Benniston, A. C.; Copley, G.; Elliott, K. J.; Harriman, A.; Stewart, B.; Zhi, Y. G. A Molecular Rotor Based on an Unhindered Boron Dipyrromethene (Bodipy) Dye. Chem. Mater. 2008, 20, 4024-4032.

(48) Bannwarth, W.; Schmidt, D.; Stallard, R. L.; Hornung, C.; Knorr, R.; Muller, F. Bathophenanthroline Ruthenium(II) Complexes as Non-Radioactive Labels for Oligonucleotides Which can be Measured by Time-Resolved Fluorescence Techniques. Helv. Chim. Acta 1988, 71, 2085.

(49) Haefele, A.; Retailleau, P.; Ziessel, R. Carbonyl Derivatives of Boradiazaindacene via Catalytic CO Insertion. J. Org. Chem . 2012, 77, 5036.

(50) Knorr, R.; Trzeciak, A.; Bannwarth, W.; Gillssen, D. New Coupling Reagents in Peptide Chemistry. Tetrahedron Lett. 1989, 30, 1927.

(51) Strickler, S. J.; Berg, R. A. Relationship between Absorption Intensity and Fluorescence Lifetime of Molecules. J. Chem. Phys. 1962, 37, 814-822. 
(52) Krueger, B. P.; Scholes, G. D.; Fleming, G. R. Calculation of Couplings and Energy-Transfer Pathways Between the Pigments of LH2 by the Ab Initio Transition Density Cube Method. J. Phys. Chem. A 1998, 102, 5378-5386.

(53) Hedley, G. J.; Ruseckas, A.; Benniston, A. C.; Harriman, A.; Samuel, I. D. W. Ultrafast Electronic Energy Transfer Beyond the Weak Coupling Limit in a Proximal but Orthogonal Molecular Dyad J. Phys. Chem. A 2015, 119, 12665-12671.

(54) Wiesenhofer, H.; Beljonne, D.; Scholes, G. D.; Hennebicq, E.; Bredas, J. L.; Zojer, E. Limitations of the Förster Description of Singlet Exciton Migration: The Illustrative Example of Energy Transfer to Ketonic Defects in Ladder-Type Poly(para-phenylenes). Adv. Funct. Mater. 2005, 15, 155-16o.

(55) Caprasecca, S.; Curutchet, C.; Mennucci, B. Quantum Mechanical Study of the Solvent-Dependence of Electronic Energy Transfer Rates in a Bodipy Closely-Spaced Dyad. Photochem. Photobiol. Sci. 2011, 10, 1602-1609.

(56) Dale, R. E.; Eisinger, L.; Blumberg, W. E. Orientational Freedom of Molecular Probes - Orientation Factor in Intramolecular Energy Transfer. Biophys. J. 1979, 26, 161-193.

(57) Denis, J. C.; Schumacher, S.; Hedley, G. J.; Ruseckas, A.; Morawska, P. O.; Wang, Y.; Allard, S.; Scherf, U.; Turnbull, G. A.; Samuel, I. D. W.; Galbraith, I. Subpicosecond Exciton Dynamics in Polyfluorene Films from Experiment and Microscopic Theory. J. Phys. Chem. C 2015, 119, 9734-9744.

(58) Hedley, G. J; Ruseckas, A.; Harriman, A.; Samuel, I. D. W. Conformational Effects on the Dynamics of Internal Conversion in Boron Dipyrromethene Dyes in Solution. Angew. Chem., Int. Ed. 2011, 50, 6634-6637.

(59) Varnavski, O.; Samuel, I. D. W.; Palsson, L. O.; Beavington, R.; Burn, P. L.; Goodson, T. Investigations of Excitation Energy Transfer and Intramolecular Interactions in a Nitrogen Corded Distrylbenzene Dendrimer System. J. Chem. Phys. 20o2, 116, 88938903.

(6o) Montgomery, N. A.; Hedley, G. J.; Ruseckas, A.; Denis, J. C.; Schumacher, S.; Kanibolotsky, A. L.; Skabara, P. J..; Galbraith, I.; Turnbull, G. A.; Samuel, I. D. W. Dynamics of Fluorescence Depolarisation in Star-Shaped Oligofluorene-Truxene Molecules. Phys. Chem. Chem. Phys. 2012, 14, 9176-9184.

(61) Atwood, P. D.; Akyant, A.; Shalumova, T.; Tanski, J. M..; Folmer-Andersen, J. F. Structural Studies of DiaminocyclohexaneContaining Aza-Crown Ether Macrocycles and Their Zn(II) Complexes. Polyhedron 2014, 67, 191-198.

(62) Fischer, G. Chemical Aspects of Peptide Bond Isomerisation. Chem. Soc. Rev. 200o, 29, 119-127.

(63) Deniz, A. A.; Dahan, M.; Grunwell, J. R.; Ha, T. J.; Faulhaber, A. E.; Chemla, D. S.; Weiss, S.; Schultz, P. G. Single-Pair Fluorescence Resonance Energy Transfer on Freely Diffusing Molecules: Observation of Förster Distance Dependence and Subpopulations. Proc. Natl. Acad. Sci. 1999, 96, 3670-3675.

(64) Beljonne, D.; Curutchet, C.; Scholes, G. D.; Silbey, R. J. Beyond Förster Resonance Energy Transfer in Biological and $\mathrm{Na}-$ noscale Systems. J. Phys. Chem. B 2009, 113, 6583-6599.

(65) Nano, A.; Retailleau, P.; Hagon, J. P.; Harriman, A.; Ziessel, R. A Hybrid Bis(amino-styryl) Substituted Bodipy Dye and its Conjugate Diacid: Synthesis, Structure, Spectroscopy and Quantum Chemical Calculations. Phys. Chem. Chem. Phys. 2010, 9, 960-967.

(66) Harriman, A.; Ziessel, R. Exploring the Effects of Solvent Polarity on the Rate of Förster-Type Electronic Energy Transfer in a Closely-Spaced Molecular Dyad. Photochem. Photobiol. Sci. 2010, 9, 960-967.

(67) Wolffs, M.; Delsuc, N.; Veldman, D.; Van Anh, N.; Williams, R. M.; Meskers, S. C. J.; Janssen, R. A. J.; Huc, I.; Schenning, A. P. H. J. Helical Aromatic Oligoamide Foldamers as Organizational Scaffolds for Photoinduced Charge Transfer. J. Am. Chem. Soc. 2009, 131, 4819-4829.
(68) Olivier, J. H.; Barbera, J.; Bahaidarah, E.; Harriman, A.; Ziessel, R. Self-Assembly of Charged Bodipy Dyes To Form Cassettes That Display Intracomplex Electronic Energy Transfer and Accrete into Liquid Crystals. J. Am. Chem. Soc. 2012, 134, 61006103.

(69) Alamiry, M. A. H; Harriman, A.; Mallon, L. J.; Ulrich, G.; Ziessel, R. Energy- and Charge-Transfer Processes in a PeryleneBODIPY-Pyridine Tripartite Array. Eur. J. Org. Chem. 2oo8, 27742782.

(70) Sahoo, H.; Nau, W. M. Phosphorylation-Induced Conformational Changes in Short Peptides Probed by Fluorescence Resonance Energy Transfer in the 10 Å domain. ChemBioChem 2007, $8,567-573$

(71) Moglich, A.; Joder, K.; Kiefhaber, T. End-to-End Distance Distributions and Intrachain Diffusion Constants in Unfolded Polypeptide Chains Indicate Intramolecular Hydrogen Bond Formation. Proc. Natl. Acad. Sci. 2006, 103, 12394-12399.

(72) Balaban, T. S.; Berova, N.; Drain, C. M.; Hauschild, R.; Huang, X. F.; Kalt, H.; Lebedkin, S.; Lehn, J. M.; Nifaitis, F.; Pescitelli, G.; Prokhorenko, V. I.; Riedel, G.; Smeureanu, G.; Zeller, J. Syntheses and Energy Transfer in Multiporphyrinic Arrays SelfAssembled with Hydrogen-Bonding Recognition Groups and Comparison with Covalent Steroidal Models. Chem. Eur. J. 2007, 13, 8411-8427.

(73) Olaya-Castro, A.; Scholes, G. D. Energy Transfer from Förster-Dexter Theory to Quantum Coherent Light-Harvesting. Int. Rev. Phys. Chem. 2011, 30, 49-77.

(74) Wong, K. F.; Bagchi, B.; Rossky, P. J.; Distance and Orientation Dependence of Excitation Transfer Rates in Conjugated Systems; Beyond Förster Theory. J. Phys. Chem. 2004, 108, 57525763.

(75) Hsu, C. P.; You, Z. Q.; Chen, H. C. H. Characterization of the Short-Range Couplings in Excitation Energy Transfer. J. Phys. Chem. C 2008, 112, 1204-1212.

(76) Khan, Y. R.; Dykstra, T. E.; Scholes, G. D. Exploring the Förster Limit for a Small FRET Pair. Chem. Phys. Lett. 20o8, 461, 305-309.

(77) Knox, R. S.; van Amerongen, H. Refractive Index Dependence of the Förster Resonance Excitation Transfer Rate. J. Phys. Chem. B 2002, 106, 5289-5293.

(78) Yeow, E. K. L.; Steer, R. P. Dynamics of Electronic Energy Transfer from the S2 State of Azulene to the S2 State of Zinc Porphyrin. Phys. Chem. Chem. Phys. 2003, 5, 97-105.

(79) Harriman, A.; Izzet, G.; Ziessel, R. Rapid Energy Transfer in Cascade-Type BODIPY Dyes. J. Am. Chem. Soc. 2oo6, 128, 10868-10875.

(8o) Wu, P. G.; Brand, L. Orientation Factor in Steady-State and Time-Resolved Resonance Energy Transfer Measurements. Biochem. 1992, 31, 7939-7947.

(81) Maineult, W.; Pelle, B.; Faoro, R.; Arimondo, E.; Pillet, P.; Cheinet, P. Dipole-Quadrupole Förster Resonance in Cesium Rydberg Gas. J. Phys. B At. Mol. Opt. Phys. 2016, 49, 214001.

(82) Blanchard-Desce, M.; Wortmann, R.; Lebus, S.; Lehn, J. M.; Kramer, P. Intramolecular Charge-Transfer in Elongated DonorAcceptor Conjugated Polyenes. Chem. Phys. Lett. 1995, 243, 526532.

(83) Czikklely, V.; Forsterling, H. D.; Kuhn, H. Extended Dipole Model for Aggregates of Dye Molecules. Chem. Phys. Lett. 1970, 6, 207-210.

(84) McDermott, G.; Prince, S. M.; Freer, A. A.; Hawthornthwaite-Lawless, A. M.; Papiz, M. Z.; Cogdell, R. J.; Isaacs, N. W. Crystal Structure of an Integral Membrane Light-Harvesting Complex from Photosynthetic Bacteria, Nature 1995, 374, 517-521.

(85) Zirzlmeier, J.; Lehnherr, D.; Coto, P. B.; Chernick, E. T.; Casillas, R.; Basel, B. S.; Thoss, M.; Tykwinska, R. R.; Guldi, D. M. Singlet Fission in Pentacene Dimers. Proc. Natl. Acad. Sci. USA 2015, 112, 5325-5330. 
(86) Sakuma, T.; Sakai, H.; Araki, Y.; Mori, T.; Wada, T.; Tkachenko, N. V.; Hasobe, T. Long-Lived Triplet Excited States of Bent-Shaped Pentacene Dimers by Intramolecular Singlet Fission.

J. Phys. Chem. A 2016, 120, 1867-1875. 
Table of Contents Graphic

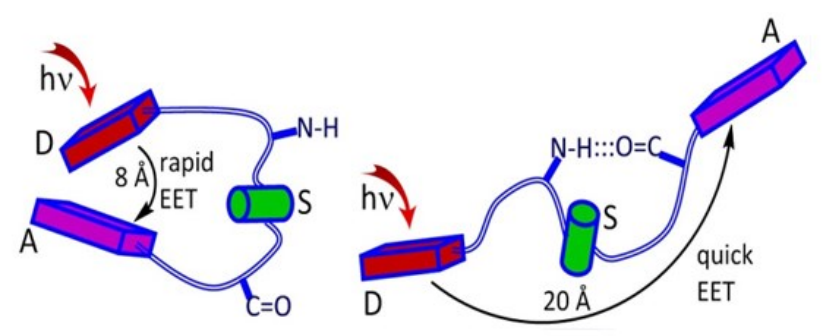

